\title{
Accuracy of pelvic multiparametric MRI in diagnosing local recurrence following radical prostatectomy. Case report and revision of the literature
}

\author{
Pietro Pepe ${ }^{1}$, Antonio Garufi ${ }^{2}$, Giandomenico Priolo ${ }^{2}$, Michele Pennisi ${ }^{1}$ \\ ${ }^{1}$ Urology Unit, Cannizzaro Hospital, Catania, Italy; \\ 2 Imaging Department, Cannizzaro Hospital, Catania, Italy.
}

\begin{abstract}
Summary A Caucasian man (73 years old) six years from radical prostatectomy for prostate cancer (PCa) showed biochemical recurrence (BCR); the follow up based on PSA evaluated every 6 months was negative $(0.1 \mathrm{ng} / \mathrm{ml})$ for 5 years, but in the last year PSA increased to $0.3 \mathrm{vs} 0.5 \mathrm{ng} / \mathrm{ml}$. The patient was asymptomatic and underwent 3.0 Tesla mpMRI equipped with surface 16 channels phased-array coil placed around the pelvic area; multiplanar turbo spin-echo T2-weighted (T2W), axial diffusion weighted imaging (DWI), axial dynamic contrast enhanced (DCE) and spectroscopy were performed. Pelvic mpMRI demonstrated the presence of a nodular tissue with a diameter of $10 \mathrm{~mm}$. located on the left of the prostatic fossa near the rectum that was higly sospicious for local PCa recurrence.

The patient underwent salvage RT (64Gy); one year from RT PSA was $0.1 \mathrm{ng} / \mathrm{ml}$ suggesting that the patient was free from recurrence.

In conclusion, mpMRI could be combined with PSA kinetics in the evaluation of men with BRC also in the presence of PSA values < $1 \mathrm{ng} / \mathrm{ml}$.
\end{abstract}

KEY WORDS: mpMRI and prostate cancer; mpMRI and local $P C a$ recurrence; $m p M R I$ and PSA failure.

Submitted 16 August; Accepted 30 September

\section{INTRODUCTION}

Despite the current stage migration in the landscape of prostate cancer (PCa), 10\%-53\% of patients will have biochemical recurrence (BCR) following radical prostatectomy (RP) as determined by serum prostate-specific antigen (PSA) measurement and PSA kinetic values (1). Since management strategies for BCR vary based on the likelihood of local versus distant recurrence, nomograms have been developed to predict the site of recurrence and likely response to localized therapy (2); given the potential side effects to which patients may be exposed with salvage therapies, such as radiation therapy (RT) (3) accurate prediction of the location of recurrence is important for selection of appropriate candidates and guidance of radiation therapy delivery.

In current practice, imaging or pathological evidence of local recurrence is not necessary to initiate local salvage treatment because current imaging techniques cannot adequately detect small-sized local recurrence. Recently, an increasing number of studies have been published reporting the acceptable diagnostic accuracy of mpMRI for detecting PCa (4) and local recurrence (5).

In this study, a case of local PCa recurrence detected by mpMRI in a man with very low PSA value is reported.

\section{Case report}

A Caucasian man (73 years old) six years before underwent RP for PCa and definitive specimen showed an organ confined cancer ( $\mathrm{pT} 2 \mathrm{CN}$ ) with focal positive surgical margin at the apex, Gleason score $7(4+3)$ and negative nodes (0/15). Follow up based on PSA value evaluated every 6 months was negative $(0.1 \mathrm{ng} / \mathrm{ml})$ for 5 years; in the last year PSA increased to $0.3 \mathrm{ng} / \mathrm{ml}$ and three months later to 0.5 $\mathrm{ng} / \mathrm{ml}$; digital rectal examination was negative. The patient was asymptomatic and underwent 3.0 Tesla mpMRI (ACHIEVA 3T; Philips Healthcare Best, the Netherlands) equipped with surface 16 channels phased-array coil placed around the pelvic area with the patient in supine position; multiplanar turbo spin-echo T2-weighted (T2W), axial diffusion weighted imaging (DWI), axial dynamic contrast enhanced (DCE) after injection of gadobutrol 0.1 $\mathrm{ml} / \mathrm{kg}$ and spectroscopy were performed (4). Pelvic mpMRI demonstrated the presence of a nodular tissue with a diameter of 10 millimeters $(\mathrm{mm})$ located on the left of the prostatic fossa near the rectum (Figure 1); moreover, the lesion showed enhancement after gadobutrol administration (DCE) resulting higly sospicious for PCa recurrence. The patient underwent pelvic salvage RT (64 Gy) and 3 months following therapy PSA was $0.1 \mathrm{ng} / \mathrm{ml}$ and no side effects were recorded. After 1 year of follow up PSA value is stable and equal to $0.1 \mathrm{ng} / \mathrm{ml}$; moreover, the patient was not submitted to additional imaging procedures or salvage therapy.

\section{Discussion}

BCR following RP develops in about $50 \%$ and $10 \%$ of high and low risk patients within 15 years from surgery. After RP, serum PSA value should decrease to an undetectable level $(<0.1 \mathrm{ng} / \mathrm{ml})$ within 30 days and should remain undetectable; according to European Association of Urology guidelines, BCR after RP is defined by two con-

No conflict of interest declared. 
Figure 1.

A man submitted to radical prostatectomy showed six years later a biochemical recurrence (PSA value equal to $0.5 \mathrm{ng} / \mathrm{ml}$ ). Multiparametric pelvic MRI demonstrated a lesion with a diameter of $10 \mathrm{~mm}$ (ring) located on the left of the rectum and provided of enhancement after gadobutrol administration that was highly suspicious for local prostate cancer recurrence.

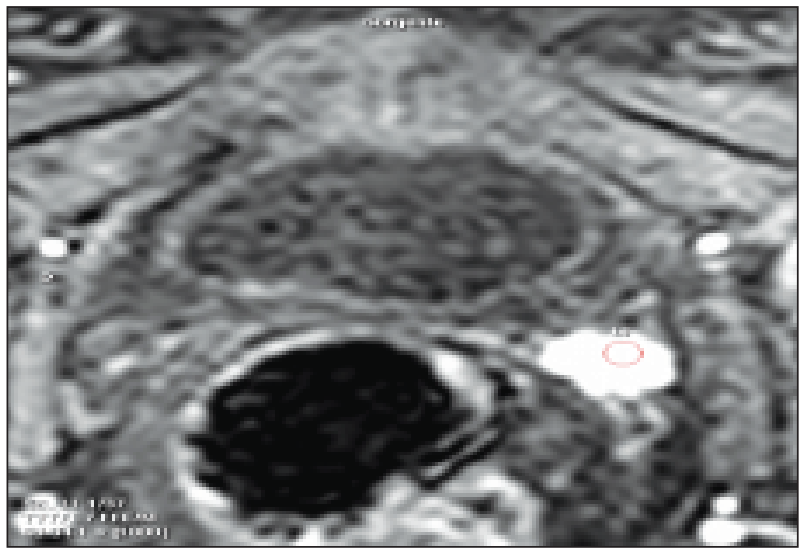

secutive values of serum PSA $>0.2 \mathrm{ng} / \mathrm{ml}$. Once that BCR occurs, the key question remains whether a PSA rise is reflective of local or distant disease in order to plan the most appropriate treatment. Generally, PSA detectable after 1 year and PSA doubling time $>10$ months are related to higher risk of local relapse; however, in the clinical practice, it is not so easy to identify the origin of the PSA relapse and sometimes many risk factors for both local and distant recurrence are present in the same patient. Moreover, it should be taken into account that the PSA level does not always correlate well with the tumour burden when are poorly differentiated. Therefore, in patients with BCR after surgical treatment, a diagnostic imaging procedure $(6,7)$ is often carried out to distinguish between local cancer recurrence and distant spread of disease. In the absence of systemic metastases salvage external RT could be assumed to be the first line treatment offering a potential chance of cure; in fact, salvage RT should be initiated when PSA levels is $<1.0 \mathrm{ng} / \mathrm{ml}$; however, the general feeling is that the lower the PSA level at the time of salvage RT, the better the result and some recent reports suggest that results are best when the serum PSA level is $<0.5 \mathrm{ng} / \mathrm{ml}$.

In the last years, mpMRI has proven to be the most useful tool available up to now for the detection and localization of local PCa recurrence after RP (6-10); Cirillo et al. (8) showed in 72 patients with BCR and PSA included between 0.2-8.8 ng/ml that mpMRI had a sensitivity, specificity, PPV (positive predictive value), NPV (negative predictive value) and accuracy in detecting locoregional relapse equal to 84.1, 89.3 , 92.5, 78.1, and 86.1\%. Panebianco et al. (9) demonstrated that mpMRI was provided of an higher accuracy in comparison with 18F-Cho PET/CT in identifying local recurrence of small lesions (range: 5-19.4 mm) and low PSA values (PSA included between 0.2 and $2.5 \mathrm{ng} / \mathrm{ml}$ ); mPMRI vs PET-CT showed a sensitivity, specificity, PPV, NPV and accuracy equal to $92,75,96,60$ and $89 \%$ vs 62 , $50,88,18$ and $60 \%$, respectively. Panebianco et al. (10) in order to validate the role of $3.0 \mathrm{~T}$ mpMRI in the detection of local PCa recurrence analyzed 126 patients with low PSA values (range: $0.5-1.7 \mathrm{ng} / \mathrm{ml}$ ) and a lesion size (range; 4-8 $\mathrm{mm}$ ); mpMRI demonstrated a sensitivity, specificity, PPV, NPV and accuracy equal to 98\%, 94\%, 97\%, 96\% and $93 \%$, respectively.

In our case, the patient had a BCR characterized by low PSA level $(0.5 \mathrm{ng} / \mathrm{ml})$ and a lesion diameter of $10 \mathrm{~mm}$; mpMRI showed the presence of PCa recurrence in the prostatic fossa allowing to perform salvage RT and PSA value one year from RT is equal to $0.1 \mathrm{ng} / \mathrm{ml}$ suggesting that the patient is free from recurrence.

In definitive, mpMRI could be combined with PSA kinetics in the evaluation of men with BRC also in the presence of PSA values $<1 \mathrm{ng} / \mathrm{ml}$.

\section{REFERENCES}

1. Cooperberg MR, Broering JM, Carroll PR. Time trends and local variation in primary treatment of localized prostate cancer. J Clin Oncol. 2010; 28:1117-1123.

2. Pound CR, Partin AW, Eisenberger MA, et al. Natural history of progression after PSA elevation following radical prostatectomy. JAMA. 1999; 281:1591-1597.

3. Peterson JL, Buskirk SJ, Heckman MG, et al. Late toxicity after postprostatectomy salvage radiation therapy. Radiother Oncol. 2009; 93:203-206.

4. Pepe P, Garufi A, Priolo G, Pennisi M. Can 3 Tesla pelvic phasedarray MRI avoid unnecessary repeat prostate biopsy in patients with PSA below $10 \mathrm{ng} / \mathrm{ml}$ ? Clinical Genitourinary Cancer. 2015; 13:e27-30.

5. Wassberg C, Akin O, Vargas HA, et al. The incremental value of contrast-enhanced MRI in the detection of biopsy-proven local recurrence of prostate cancer after radical prostatectomy: effect of reader experience. Am J Roentgenol. 2012; 199:360-366.

6. Garcia JR, Romera N, Cozar Met al. (11)C-choline PET/CT and multiparametric MRI in patients with biochemical relapse of prostate cancer. Actas Urol Esp. 2015; 39:259-263.

7. Barchetti F, Panebianco V. Multiparametric MRI for recurrent prostate cancer post radical prostatectomy and postradiation therapy. Biomed Res Int. 2014; 2014:316272.

8. Cirillo S, Petracchini M, Scotti L, et al. Endorectal magnetic resonance imaging at 1.5 Tesla to assess local recurrence following radical prostatectomy using T2-weighted and contrast-enhanced imaging. Eur Radiol. 2009; 19:761-769.

9. Panebianco V, Sciarra A, Lisi D, et al. Prostate cancer: 1HMRSDCEMR at 3T versus $[(18) F]$ choline PET/CT in the detection of local prostate cancer recurrence in men with biochemical progression after radical retropubic prostatectomy (RRP). Eur J Radiol. 2012; 81:700-708.

10. Panebianco V, Barchetti F, Sciarra A, et al. Prostate cancer recurrence after radical prostatectomy: the role of 3-T diffusion imaging in multi-parametric magnetic resonance imaging. Eur Radiol. 2013; 23:1745-1752.

\section{Correspondence}

Pietro Pepe, MD

piepepe@hotmail.com

Michele Pennisi, MD

Urology Unit, Cannizzaro Hospital

Via Messina 829, Catania, Italy

Antonio Garufi, MD

Giandomenico Priolo, MD

Imaging Department, Cannizzaro Hospital, Catania, Italy 